\title{
Loss of Fezf2 promotes malignant progression of bladder cancer by regulating the NF-KB signaling pathway
}

\author{
Zhaohui Chen $^{1,2} \cdot$ Lijie Zhou $^{1} \cdot$ Xuehan Liu $^{3} \cdot$ Longwang Wang $^{1,2} \cdot$ Gallina Kazobinka $^{1} \cdot$ Xiaoping Zhang $^{1} \cdot$ Teng Hou $^{1}$
}

Received: 8 August 2017 / Revised: 6 April 2018 / Accepted: 13 April 2018 / Published online: 20 June 2018

(c) United States \& Canadian Academy of Pathology 2018

\begin{abstract}
Forebrain embryonic zinc finger 2 (Fezf2) is an evolutionarily conserved zinc finger transcription repressor. It has been reported to be a tumor suppressor; however, neither the role that Fezf2 plays in bladder cancer nor the mechanisms involved have been investigated. In this study, we showed that Fezf2 expression is downregulated in bladder cancer tissues and cell lines compared to adjacent non-tumor tissues and normal urothelial cells. We also retrospectively analyzed the association between Fezf2 and various clinicopathologic characteristics in 196 bladder cancer patients, and showed that low expression of Fezf2 is correlated with larger tumor size, advanced tumor stage, and poor clinical prognosis. Moreover, we found that overexpression of Fezf2 significantly inhibited the proliferation, growth, migration, and invasion of bladder cancer cells, and attenuated angiogenesis, while knockdown of Fezf2 had the opposite effect. Fezf2 suppressed bladder cancer aggressiveness by activating the NF- $\mathrm{KB}$ signaling pathway. These findings suggest that Fezf 2 holds promise as a prognostic biomarker, and provide a putative mechanism for bladder cancer progression.
\end{abstract}

\section{Introduction}

Bladder cancer is one of the foremost causes of cancerrelated death in men worldwide [1]. Approximately $75 \%$ of newly diagnosed bladder cancers are non-muscle-invasive tumors and have a high survival rate. However, up to $15 \%$ of these patients will eventually progress to muscle-invasive bladder cancer (MIBC), which has a dismal long-term

These authors contributed equally: Zhaohui Chen, Lijie Zhou, and Xuehan Liu.

Electronic supplementary material The online version of this article (https://doi.org/10.1038/s41374-018-0077-9) contains supplementary material, which is available to authorized users.

Teng Hou

1aiyan@163.com

1 Department of Urology, Union Hospital, Tongji Medical College, Huazhong University of Science and Technology, Wuhan 43002, China

2 Department of Urology, The Second Affiliated Hospital of Nanchang University, Nanchang 33000, China

3 Department of Epidemiology and Biostatistics and State Key Laboratory of Environment Health, School of Public Health, Tongji Medical College, Huazhong University of Science and Technology, Wuhan 43003, China prognosis. For patients with advanced or metastatic disease, the median survival is less than 14 months, and the longterm disease-free survival rate is $\sim 15 \%$ [2]. Therefore, clarification of the molecular mechanisms that trigger the neoplastic transformation of urothelium and progression of bladder cancer is warranted.

Forebrain embryonic zinc finger 2 (Fezf2) is a newly described gene located on chromosome $3 p 14$, which encodes an evolutionarily conserved forebrain-enriched zinc finger transcription repressor [3]. Fezf2 is expressed in the forebrain of the clawed frog (Xenopus), zebrafish and mice, and has a role in patterning the development of the diencephalon, and specifying distinct forebrain neuronal subtypes $[4,5]$. Fezf2 is linked to the regulation of neural stem cell differentiation in a significant manner [6], and has recently been reported to be dysregulated in human cancers. Shu et al. demonstrated that Fezf2 acts as a histone deacetylase-associated repressor, and is frequently downregulated in nasopharyngeal carcinoma, with a high degree $(75.5 \%)$ of promoter methylation in nasopharyngeal tumors [7]. Gan et al. also identified Fezf2 as a biomarker in welldifferentiated colorectal carcinoma (CRC), and it has been suggested that this molecule is related to the tumorigenesis, proliferation, and differentiation of CRC [8]. Denkert et al. identified differences in metabolic patterns of Fezf2 in ovarian borderline tumors and invasive ovarian carcinomas 
[9]. These studies gave rise to the hypothesis that Fezf2 plays an important role in human cancers.

The role and mechanism of Fezf2 in bladder cancer progression are unknown. In the present study, we hypothesize that Fezf2 is a suppressor of bladder cancer development. We provide the first evidence that Fezf2 expression is closely correlated with various clinicopathologic features of bladder cancer, and with clinical prognosis in bladder cancer patients, and we show evidence which argues that Fezf2 modulates cancer progression through the NF- $\mathrm{KB}$ pathway. Our findings highlight the critical role of Fezf2 as a tumor suppressor, and the potential of Fezf2 as a target for bladder cancer therapy.

\section{Material and methods}

\section{Cell culture}

Bladder cancer cell lines (BIU-87, T24, J82, UM-UC-3, RT4, EJ, and TCCSUP) and one normal urothelial cell line (SV-HUC-1) were purchased from the Cell Bank of the Chinese Academy of Sciences (Shanghai, China). Primary normal bladder urothelial cells (NBUCs) cultures and the human uroepithelial cell line SV-HUC-1 were established as described previously [10]. The cell lines BIU-87, T24, J82, UM-UC-3, EJ, and TCCSUP were maintained in RPMI 1640 medium (Gibco, USA) supplemented with $10 \%$ fetal bovine serum (FBS) (Gibco, USA), and the RT4 cell line was cultured in McCoy's 5a medium supplemented with $10 \%$ FBS. SV-HUC-1 cells were grown in F12K medium supplemented with $10 \%$ FBS.

\section{Tissue specimens}

One hundred and ninety-six paraffin-embedded bladder cancer tissue blocks were selected and evaluated retrospectively for this study. These archived specimens had originally been histopathologically and clinically diagnosed at the Second Affiliated Hospital of Nanchang University. The staging of all samples was determined according to the American Joint Committee on Cancer (AJCC) classification system on TNM staging. Tumor grade was determined according to the World Health Organization/ International Society of Urological Pathologists criteria. Transurethral resection was performed in patients with non-muscleinvasive cancer, and radical cystectomy was performed in patients with muscle-invasive cancer, according to the National Comprehensive Cancer Network (NCCN) guidelines. Patient consent for this study was obtained in each case, and the study was approved by the ethics committee of the Second Affiliated Hospital of Nanchang University. In addition, 60 pairs of snap-frozen bladder cancer, and samples of normal urothelium were obtained and frozen immediately in liquid nitrogen after surgical removal (cystectomy) and stored at $-80^{\circ} \mathrm{C}$ until use. To select adjacent non-tumor bladder tissues, grossly normal mucosa from the resection margin farthest from tumor was excised and subjected to frozen section evaluation in order to exclude dysplasia or presence of carcinoma cells. The urothelium and submucosal layers of the adjacent area was then carefully peeled off and placed immediately in liquid nitrogen.

\section{Gene set enrichment analysis}

The expected relative mRNA expression of Fezf2 in bladder tumor vs. normal urothelium samples was obtained from the National Center for Biotechnology Information (NCBI) gene expression omnibus database (NCBI accession no. GSE3167). Correlation between Fezf2 expression and NF$\kappa \mathrm{B}$ signaling pathway signatures was analyzed using the publicly available bladder cancer patient expression profiles obtained from The Cancer Genome Atlas (TCGA, https://ca ncergenome.nih.govl) and application of gene set enrichment analysis (GSEA) [11].

\section{EdU labeling}

Cells were incubated with 5-ethynyl-2'-deoxyuridine (EdU, RiboBio; R11053) for $3 \mathrm{~h}$ at $37^{\circ} \mathrm{C}$, and treated with ApolloR reaction cocktail according to the manufacturer's instructions. Images were collected using fluorescent microscopy (Olympus, Japan).

\section{Flow cytometry analysis}

Cells were harvested, washed, fixed with $75 \%$ alcohol, and incubated with $2 \mu \mathrm{g} / \mathrm{ml}$ bovine pancreatic RNAase (SigmaAldrich) at $37{ }^{\circ} \mathrm{C}$ for $30 \mathrm{~min}$. Afterward, these cells were incubated in $20 \mathrm{mg} / \mathrm{ml}$ of propidium iodide (Sigma-Aldrich; USA) at room temperature for $20 \mathrm{~min}$. The cell cycle was analyzed by using a BD LSRFortessa X-20 instrument (BD Biosciences, CA, USA).

\section{Invasion assay}

The capacity for cell invasion was evaluated with transwell chambers (Corning LifeSciences). After pretreatment, $1 \times$ $10^{5}$ cells suspended in $100 \mathrm{ml}$ of serum-free medium were seeded in each upper pre-coated Matrigel transwell insert chamber, and culture medium supplemented with $10 \%$ FBS was added to the lower chamber. After incubation for $24 \mathrm{~h}$, the cells remaining on the upper surface of the membrane were removed and cells that had migrated to the lower surface of the membrane were fixed and stained with $0.1 \%$ crystal violet. 


\section{Chick chorioallantoic membrane assay}

Fertilized chicken eggs were obtained from the Institutional Animal Care and Use Committee of Tongji Medical College at Huazhong University of Science and Technology. Ethics approval was obtained from the Huazhong University of Science and Technology Animal Ethics Committee. A window $1.0 \mathrm{~cm}$ in diameter was created in the egg shell on day three of chick embryo development, and the shell membrane was humidified with phosphate buffer solution (PBS) and carefully removed. The window was resealed with adhesive tape and the eggs were returned to the incubator until the day of the experiment. On day seven, $5 \times 10^{6}$ tumor cells were mixed with growth factor reduced matrigel $(8.9 \mathrm{mg} / \mathrm{mL}$, BD Biosciences, CA, USA). Matrigel grafts were placed on top of the chick chorioallantoic membrane (CAM) and the eggs were sealed and incubated at $37^{\circ} \mathrm{C}$ with $70 \%$ relative humidity for four days. A mixture of methanol and acetone (1:1 by volume) was then added directly to immerse and fix the blood vessels of the experiment zone. Finally, the CAM was separated from the embryo, cut and spread on a glass slide, and gross photos of each CAM were taken with a digital camera (Canon, Japan). The eggs were placed on ice for $4 \mathrm{~h}$ to euthanize the chick embryos by hypothermia.

\section{Human umbilical vein endothelial cell tube formation assay}

A volume of $200 \mu \mathrm{l}$ of precooled Matrigel (Collaborative Biomedical Products) was pipetted into each well of a 24well plate and incubated for $30 \mathrm{~min}$ at $37^{\circ} \mathrm{C}$. Human umbilical vein endothelial cells (HUVECs) $\left(5 \times 10^{4}\right)$ in 200 $\mu \mathrm{l}$ conditioned medium were added to each well and incubated at $37{ }^{\circ} \mathrm{C}$ in $5 \% \quad \mathrm{CO}_{2}$ for $20 \mathrm{~h}$. The capillary tube structure was photographed under a $100 \times$ bright-field microscope, and quantified by measuring the total length of the completed tubes. Each condition was assessed at least in triplicate.

\section{Quantitative real-time PCR}

Total RNA from tissue samples and cells was extracted using the TRIzol reagent kit (Invitrogen, California, USA). Reverse transcription-PCR was performed using the RevertAid First Strand cDNA Synthesis kit (Thermo, Massachusetts, USA). Real-time quantitative PCR was performed on a StepOne Plus real-time PCR system (Life Technologies, Carlsbad, CA, USA). The sequences of primers are provided in Supplementary Table 1. Glyceraldehyde 3-phosphate dehydrogenase (GAPDH) was used as an internal control.

\section{Western blotting}

Cells and tissues were lysed with RIPA Lysis Buffer, and cleared by centrifugation at $4{ }^{\circ} \mathrm{C}$. The protein concentration of each lysate was determined with the Bradford assay (Thermo Scientific, Massachusetts, USA). Equal amounts of protein were separated by SDS-PAGE gels, and then transferred to polyvinylidene difluoride membrane, and probed with corresponding antibodies: Fezf2 (Abcam, cat. no. ab69436), anti-p-IкB $\alpha$ (Cell Signaling Technology, cat. no. 2859), anti-IкB $\alpha$ (Cell Signaling Technology, cat. no. 4814), anti-p-IKK $\beta$ (Cell Signaling Technology, cat. no. 2078), anti-IKK $\beta$ (Cell Signaling Technology, cat. no. 2370), anti-p65 (Cell Signaling Technology, cat. no. 4764), anti-p84 (Abcam, cat. no. ab131268), and anti- $\alpha$-tubulin (Santa Cruz, cat. no. sc-5286).

\section{Immunohistochemistry}

Formalin-fixed, paraffin-embedded specimens were cut into 4- $\mu \mathrm{m}$ thick sections for immunohistochemical staining. Sections were deparaffinized in xylene and rehydrated, and were then treated with 3\% hydrogen peroxide in methanol to quench the endogenous peroxidase activity, followed by incubation with $1 \%$ fish skin gelatin to block nonspecific binding. Tissue sections were incubated with rabbit polyclonal anti-Fezf2 antibody (Abcam, Cambridge, USA; 1:200; cat. no. ab214186) at $4{ }^{\circ} \mathrm{C}$ overnight, and incubated with a biotinylated secondary antibody (Abcam, Cambridge, USA), followed by further incubation with 3,3diaminobenzidine tetrahydrochloride (DAB), and counterstaining with hematoxylin. Evaluation of immunohistochemical staining (IHC) was performed by two independent observers who were blinded to the patient clinical data. The quantity and quality of immunostaining was scored based both on the proportion of positively stained tumor cells and on the intensity of staining. IHC intensity was scored as 0 (no staining); 1 (weak staining $=$ light yellow); 2 (moderate staining $=$ yellow brown); or 3 (strong staining $=$ brown). The percentage of positive cells was scored as $0=$ negative; $1=10 \%$ or less; $2=11$ to $50 \% ; 3=51$ to $80 \%$; or $4=80 \%$ or more cells positive. The final score was obtained by multiplying the proportion of positive cells and the staining intensity score, and the median value was used to create dichotomous variables. A final score of $0-2$ was considered to be negative, and a final score of 3-12 was considered positive as in previous studies [12, 13].

\section{Plasmids, lentiviral infection, and transfection}

Human Fezf2 cDNA was amplified by PCR and cloned into the GV358 lentiviral vector (GeneChem, Shanghai, China). An oligo of Fezf2 shRNAs was synthesized and inserted in 
a GV248 vector Genechem Co., Ltd (GeneChem, Shanghai, China). Stable cell lines that expressed Fezf2 or Fezf2shRNA were selected at 10 days with $0.5 \mathrm{mg} / \mathrm{ml}$ puromycin.

\section{Anchorage-independent growth ability assay}

Cells were trypsinized and counted. $5 \times 10^{3}$ cells were mixed with complete medium containing $0.3 \%$ agar (Sigma-Aldrich; USA) on 6-well plates, followed by plating over a bottom layer with $1 \%$ agar complete medium mixture. After 10 days of incubation, viable colonies larger than $0.1 \mathrm{~mm}$ in diameter were scored.

\section{Tumor xenografts}

All experimental procedures were approved by the Institutional Animal Care and Use Committee of Tongji Medical College of Huazhong University of Science and Technology. BALB/c nude mice (5-6 weeks old) were purchased from the Center of Experimental Animal of Tongji Medical College of Huazhong University of Science and Technology and randomized into groups. For tumor formation assay, $5 \times 10^{6}$ cells were injected subcutaneously into one single side of each mouse. Tumor volume was calculated using the following equation: length $\times(\text { width })^{\wedge} / 2$. Mice were sacrificed after 30 days, and the tumors were evaluated by an IVIS imagining system (Caliper).

\section{Luciferase reporter assay}

Cells $\left(5 \times 10^{4}\right)$ were seeded in triplicate wells of 24-well plates and allowed to settle for $24 \mathrm{~h}$. A volume of $100 \mathrm{ng}$ of luciferase reporter plasmid or the control plasmid, together with $1 \mathrm{ng}$ pRL-TK Renilla plasmid (Promega) were cotransfected using Lipofectamine 3000 reagent (Invitrogen, USA). Luciferase and Renilla signals were measured $48 \mathrm{~h}$ after transfection by using the Dual Luciferase Reporter Assay Kit (Promega, USA) according to the manufacturer's instruction. All experiments were performed in triplicate.

\section{Statistical analysis}

All statistical analyses were carried out using SPSS 16.0 software. Differences between groups were analyzed by using the non-parametric Mann-Whitney test. The correlation between Fezf2 expression and bladder cancer clinicopathologic characteristics was analyzed using the chi-square test or Fisher's exact test. Survival curves were plotted with the Kaplan-Meier method and compared using the log-rank test. Survival data were evaluated by using multivariate Cox regression analysis. $P<0.05$ was considered statistically significant. Data are expressed as mean $\pm \mathrm{SD}$ from at least three independent experiments.

\section{Results}

\section{Downregulation of Fezf2 correlates with tumor progression and poor prognosis in bladder cancer patients}

To investigate the role of Fezf 2 expression in bladder cancer progression, we first analyzed Fezf2 expression in bladder cancer cells and tissues. In 85.0\% (51/60) of bladder cancer patients, Fezf 2 mRNA expression was decreased in bladder cancer tissues as compared to matched normal tissue (Fig. 1a). Consistent with this finding, western blot analysis showed that Fezf2 expression was markedly lower in bladder cancer than that in adjacent non-tumor tissue (Fig. 1b). In addition, Fezf2 was significantly downregulated in all 7 bladder cancer cell lines compared with NBUCs and normal urothelial cells (Fig. 1c, d). Fezf2showed notably higher expression in the RT4 cell line, which is derived from a human bladder transitional cell papilloma, than in the other six bladder cancer cell lines. Aanalysis of mRNA microarray data from the GSE3167 database showed that Fezf2 mRNA expression was dramatically decreased in bladder cancer tissues as compared to normal bladder tissues $(P<0.001$, Fig. 1e).

To determine the clinical relevance of Fezf2 in bladder cancer, Fezf2 expression was examined in 196 paraffinembedded, archived bladder cancer tissues by IHC assay (Fig. 1g). Correlation analysis of Fezf2 expression revealed a significant association between Fezf2 levels and tumor stage (T classification) $(P=0.027)$ and tumor size $(P=$ 0.036). However, there wasn't significant association of Fezf2 expression with sex, age, or tumor grade (Supplementary Table 2). Based on the Kaplan-Meier method, the mean overall and recurrence-free survival times were 50.9 and 29.5 months, respectively. It is of note that patients without Fezf2 expression had shorter overall and recurrence-free survival time $(P=0.015$ and $P=0.010$, respectively) (Fig. 1f). Multivariable Cox Regression analyses indicated that Fezf2 expression is a significant independent predictor of both $\mathrm{OS}(\mathrm{HR}=0.318, P=0.031)$ and RFS (HR $=0.462, P=0.009)$ in bladder cancer patients after adjusting for sex, age, grade, tumor size, and $\mathrm{T}$ classification (Supplementary Table 3). In order to prevent problems arising from overfitting, we performed bootstrap validation in the regression models (Supplementary Table 3), and calculated the variance inflation factors of variables in the Cox regression models (Supplementary Table 4). The results indicate that the probability of overfitting is slim. Moreover, we calculated the c-index in 
A

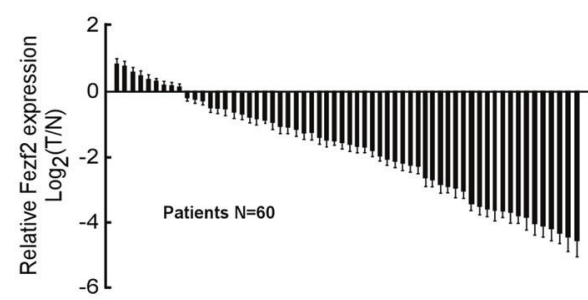

C

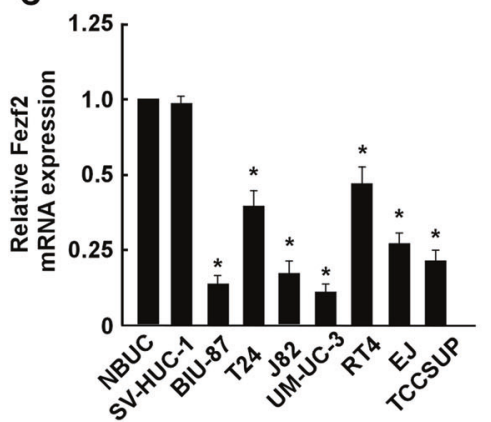

$\mathbf{F}$

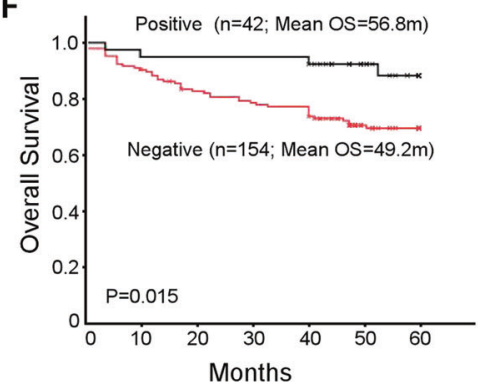

B

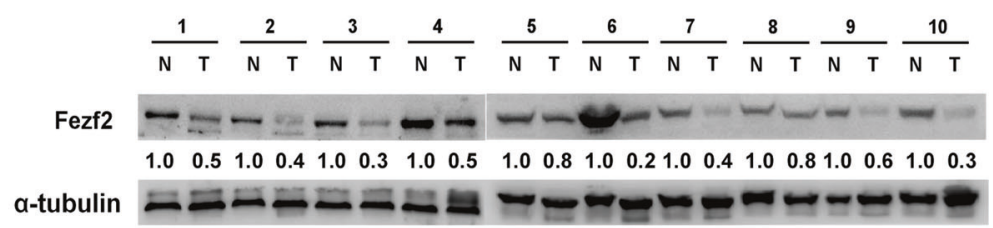

$\mathrm{E}$

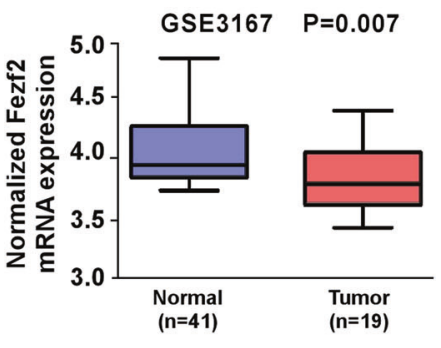

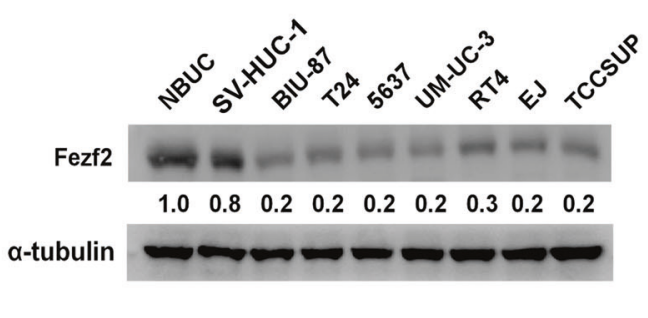

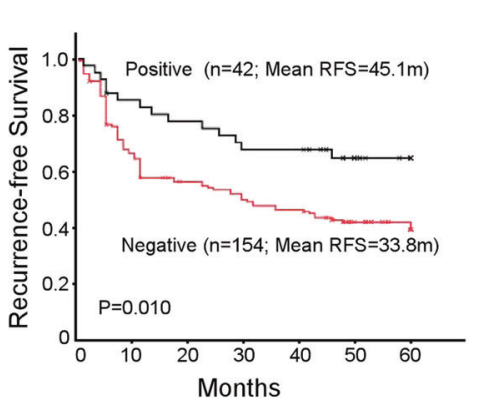

G

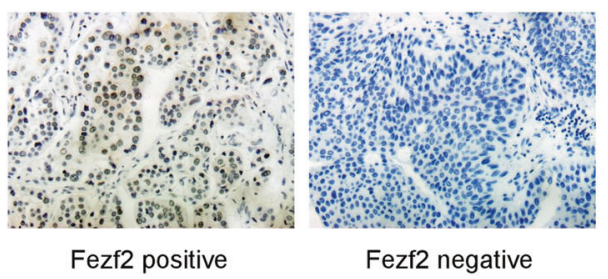

tumor and normal tissue of bladder cancer patients from GSE3167 database. f Kaplan-Meier analysis of overall survival curves for bladder cancer patients with negative vs. positive Fezf2 expression. Fezf2 positivity was significantly correlated with more favorable overall survival $(P=0.010)$ and recurrence-free survival $(P=0.015)$. OS overall survival, RFS recurrence-free survival. $\mathbf{g}$ Representative images of IHC analysis of Fezf2 in bladder cancer specimens. 400x

models with and without Fezf2, and found that the c-index was significantly increased in model with Fezf 2 compared to those without Fezf2, indicating that Fezf2 is a significant independent prognostic predictor after controlling for the other parameters. The addition of Fezf 2 improved accuracy of predicting OS by $5.6 \%(P=0.071)$ and RFS by $5.3 \%(P$ $<0.05$ ) (Supplementary Table 5). Our findings indicate Fezf2 may be a predictive biomarker of clinical outcome in bladder cancer.

\section{Overexpression of Fezf2 suppresses aggressiveness of bladder cancer cells}

To assess the biologic role of Fezf2 in bladder cancer cells, stable Fezf2-overexpressing and Fezf2-knockdown EJ and T24 cells were established (Fig. 2a). Ectopic expression of Fezf 2 drastically decreased proliferation in both cell lines
(Fig. 2b). Similarly, flow cytometry revealed that overexpression of Fezf2 significantly reduced the percentage of $\mathrm{S}$ phase cells in the sample (Fig. 2c). The anchorageindependent growth assay demonstrated that cells overexpressing Fezf2 form a smaller number of colonies and smaller sized colonies compared with control cells (Fig. 2d). In addition, Transwell assays showed Fezf2-overexpressing cells exhibit significantly decreased invasive ability (Fig. 2e). Moreover, conditioned medium from Fezf2transduced bladder cancer cells exhibited decreased ability for induction of tubule formation by HUVECs (Fig. 2f), and also showed decreased ability for induction of vessel formation in the CAM assay (Fig. 2g). Consistent with these results, silencing of Fezf2 induced cell proliferation, invasion, and angiogenesis (Fig. 3a-g). These results suggest that Fezf2 plays a significant role in the aggressiveness of bladder cancer in vitro. 
A

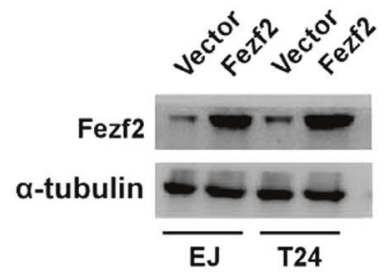

B

พื

$\stackrel{5}{\mathbb{2}}$

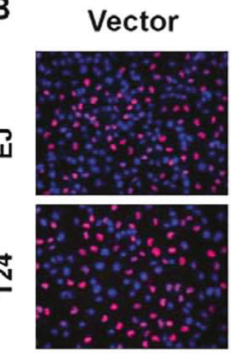

Fezf2

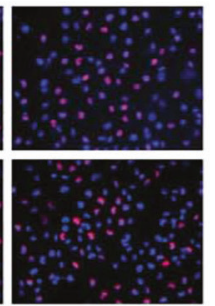

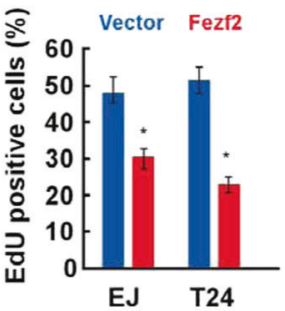

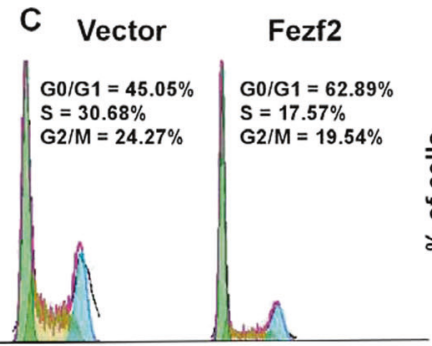

EJ
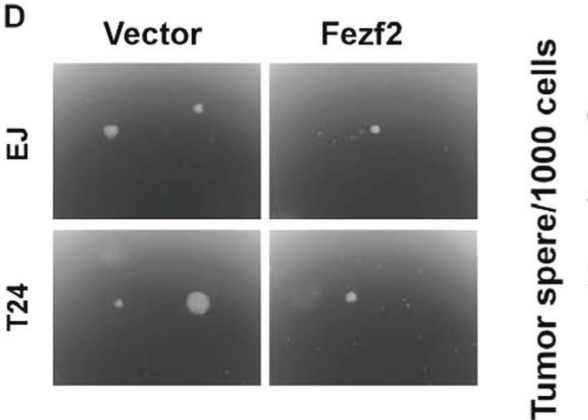

Vector Fezf2
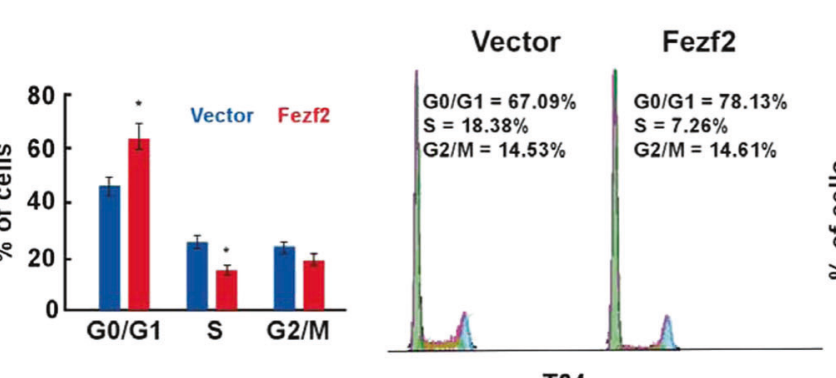

T24

E
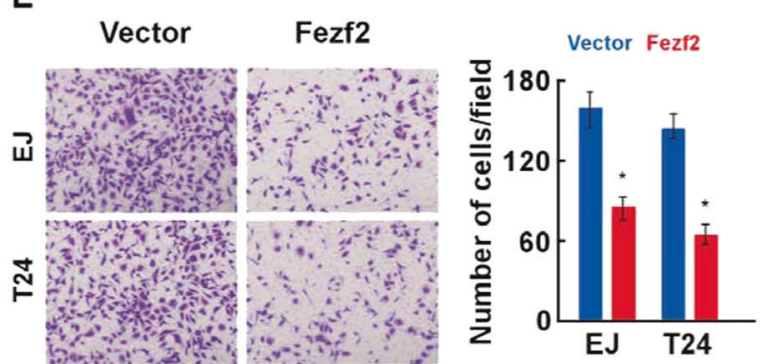

G

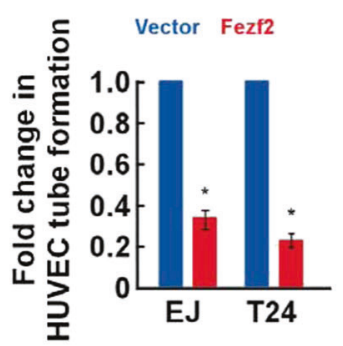

Fig. 2 Upregulation of Fezf2 inhibits bladder cancer cell aggressiveness in vitro. a Western blotting analysis of Fezf2 expression in EJ and T24 cells stably expressing Fezf2; $\alpha$-tubulin was used as a loading control. b Representative micrographs (left panel) and quantification (right panel) of EdU incorporation in indicated bladder cancer cells. DAPI was used as a DNA/nuclear stain. $\mathbf{c}$ Representative images of CAM blood vessels stimulated with conditioned medium from indicated cells. d Flow cytometric analysis showing the percentages of

\section{Overexpression of Fezf2 abolishes tumorigenicity of bladder cancer in vivo}

We next investigated the effect of Fezf2 on the tumorigenicity of bladder cancer cells in vivo. The results showed that tumor cells overexpressing Fezf 2 were significantly smaller and weighed less than control cells (Fig. 4a-c). Immunohistochemical staining showed lower expression of Ki67 and CD31, and less microvascular density in groups overexpressing Fezf2 than in the control groups (Fig. 4d). These findings indicate knockdown of Fezf2 contributes to bladder cancer tumorigenicity in vivo. 
A

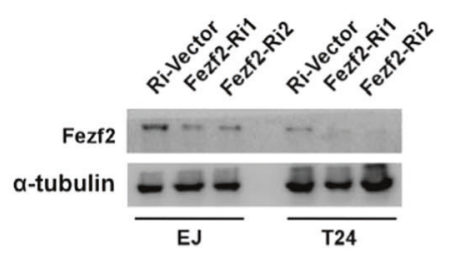

B

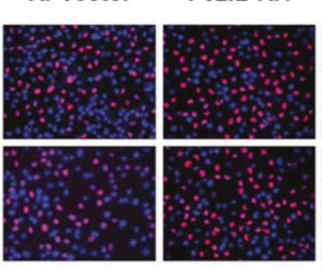

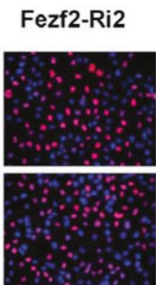

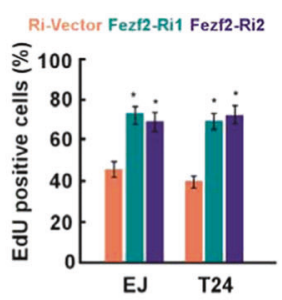

C
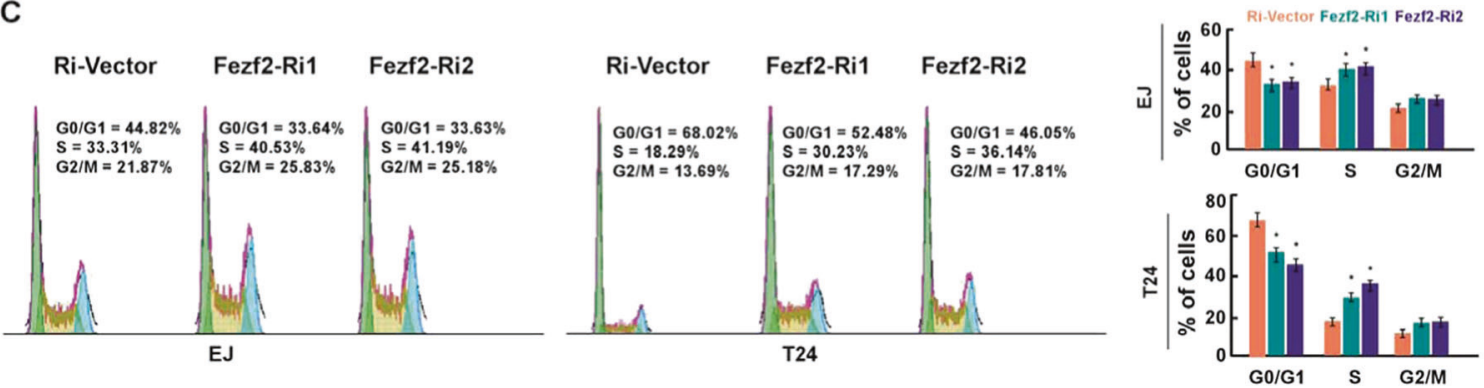

D
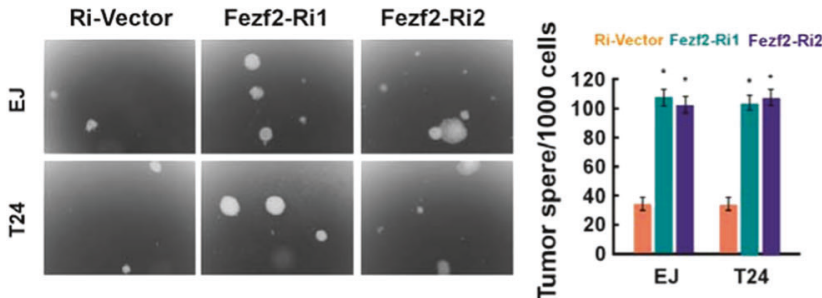

E
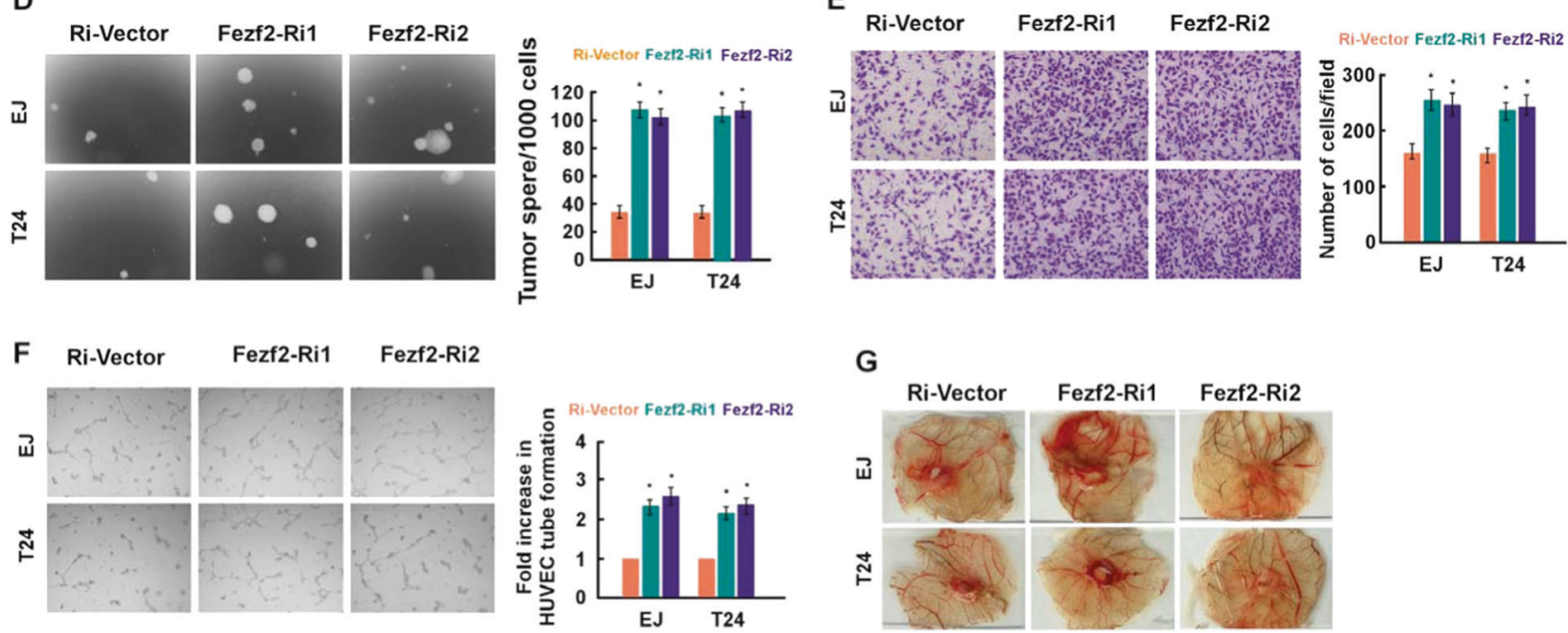

Fig. 3 Downregulation of Fezf2 promotes aggressiveness in bladder cancer cells. a Western blotting analysis of Fezf2 expression in EJ and T24 cells with stable silencing of Fezf2; $\alpha$-tubulin was used as a loading control. b Representative micrographs (left panel) and quantification (right panel) of EdU incorporation in indicated bladder cancer cells. DAPI was used as a DNA/nuclear stain. $\mathbf{c}$ Representative images of CAM blood vessels stimulated with conditioned medium from indicated cells. d Representative pictures (left panel) and

\section{Fezf2 mediates the NF-KB signaling pathway in bladder cancer cells}

To further explore the molecular mechanisms of proliferation, metastasis, and angiogenesis which are inhibited by Fezf2 in bladder cancer, GSEA of publicly available gene expression array data was analyzed, and Fezf 2 expression was found to be negatively associated with the activation of NF- $\mathrm{kB}$ signaling (Fig. 5a). Overexpression of Fezf2 significantly enhanced the activity of NF- $\mathrm{NB}$ luciferase reporter activity, whereas silencing of Fezf2 inhibited reporter

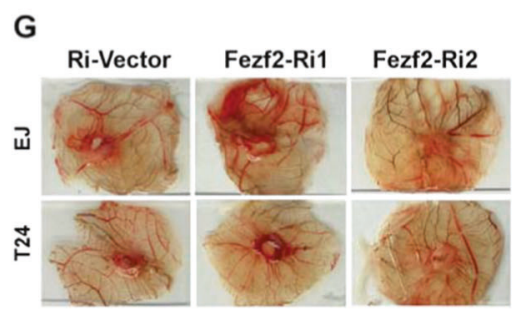

quantification (right panel) of invading cells were analyzed using a transwell Matrigel assay. e Flow cytometric analysis showing percentages of Fezf2-knockdown cells at different phases of the cell cycle. f Representative images (left panel) and quantification (right panel) of HUVECs cultured on matrigel-coated plates with conditioned medium from Fezf2-knockdown cells. Bar graphs show statistical analysis of three independent experiments $(* P<0.05)$

activity (Fig. 5b). The expression of genes downstream of NF-kB was reduced in cells overexpressing Fezf2, but expression was elevated in Fezf2-knockdown cells (Fig. 5c). Moreover, western blotting showed that expression of nuclear $\mathrm{p} 65$, phosphorylated-IKK $\beta$, and $\mathrm{p}$-I $\mathrm{KB} \alpha$ were remarkably decreased in Fezf2-overexpressing cells, but expression of these genes was increased in Fezf2knockdown cells (Fig. 5d), arguing Fezf2 suppresses the activation of the NF- $\mathrm{kB}$ signaling pathway.

To more strongly validate the pathogenesis of bladder cancer which is mediated by Fezf2 and occurs through NF- 
A

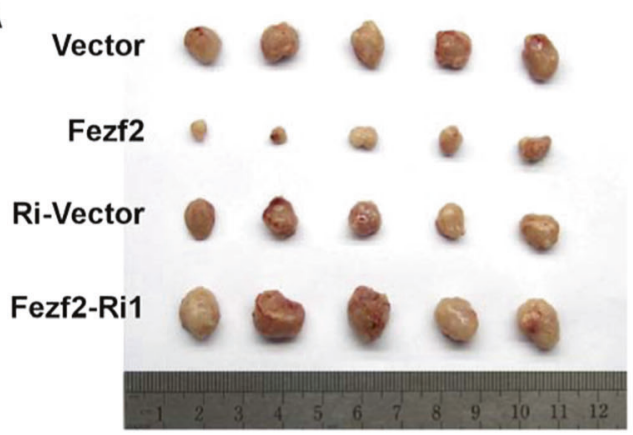

B

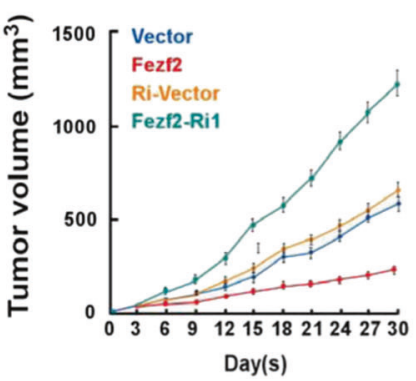

C

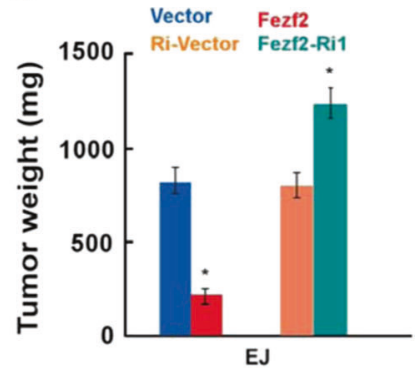

D

Vector
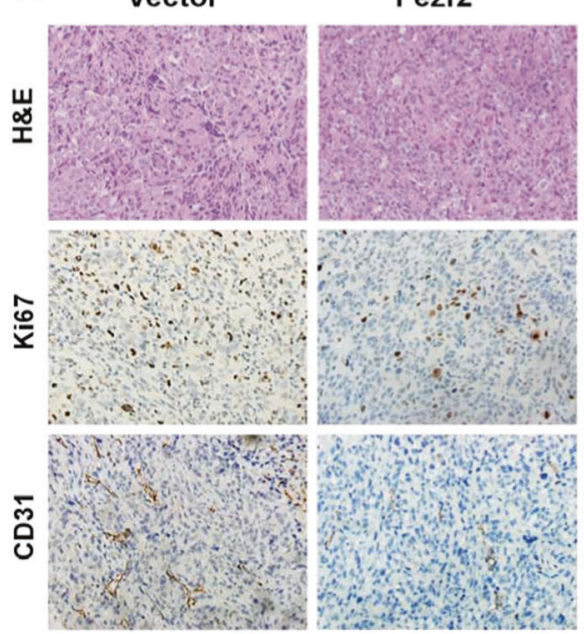

Ri-Vector
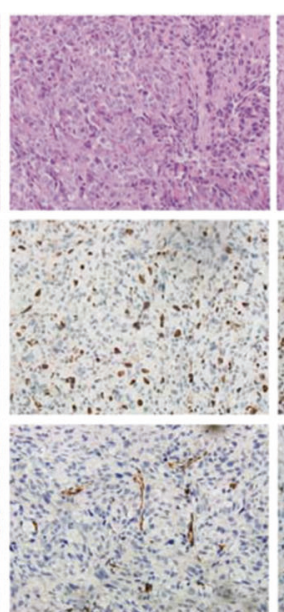

Fezf2-Ri1

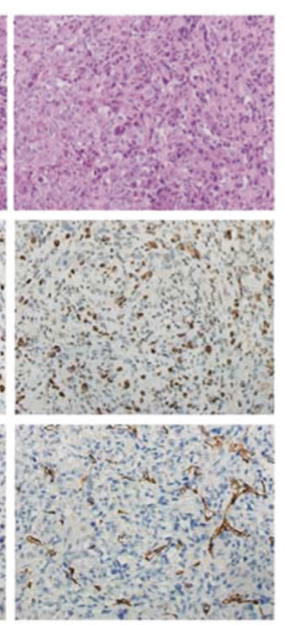

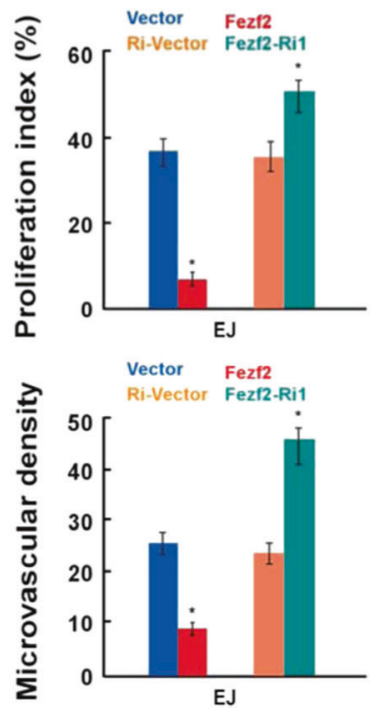

Fig. 4 Fezf 2 overexpression suppresses tumorigenicity of bladder cancer in vitro. a Representative micrographs (left) and quantification (right) of colonies formed by indicated cells, determined by anchorageindependent growth assays. b Representative images of tumors from nude mice in xenograft model $\mathbf{c}$ Tumor volumes were measured on indicated days. d Tumor weights of all mice in each group. e Immunohistochemical staining shows expression of Ki67 and CD31 in indicated tissues. Data are represented as mean \pm SD of three independent experiments. $* P<0.05$ $\kappa \mathrm{B}$ signaling, we blocked the NF- $\kappa \mathrm{B}$ pathway in Fezf2knockdown cells by overexpressing an I $\mathrm{I} B \alpha$ dominantnegative mutant (IкB $\alpha$-mut). As expected, the augmented effect of Fezf2-knockdown on NF-kB activation was significantly attenuated by transfection of the IкB $\alpha$-mut or by application of NF- $\mathrm{kB}$ inhibitor. Consistent with these findings, blockade of the NF- $\mathrm{\kappa B}$ pathway abolished the effect of Fezf2-knockdown on bladder cancer aggressiveness to a significant extent (Fig. 5e-g). These results strongly suggest Fezf 2 contributes to NF- $\mathrm{KB}$ activation, which in turn mediates aggressiveness of bladder cancer cells.

\section{Clinical association of Fezf2-mediated NF-кB activation in human bladder cancer}

To examine the clinical relevance of Fezf2 expression and NF- $\kappa B$ activation in human bladder cancer, the expression of Fezf2 and NF- $\mathrm{KB}$ downstream factors in 10 freshly prepared bladder cancer tissues using real-time PCR and western blot assays were examined. Fezf2 levels were negatively correlated with nuclear p65 signals and the expression of NF- $\mathrm{kB}$ downstream genes (Fig. 6a, b). These data further support our hypothesis that downregulation of Fezf2 inactivates the NF- $\mathrm{KB}$ signaling pathway, which consequently leads to an aggressive bladder cancer phenotype.

\section{Discussion}

In this study there were several discoveries concerning the role of Fezf2 in the malignant progression of bladder cancer. First we demonstrated that Fezf2 is significantly downregulated in bladder cancer, and this downregulation is associated with clinical features and prognosis of bladder cancer patients. Second, we showed that overexpression of Fezf 2 inhibits the proliferative and invasive abilities of bladder cancer cells, while downregulation of Fezf2 
A

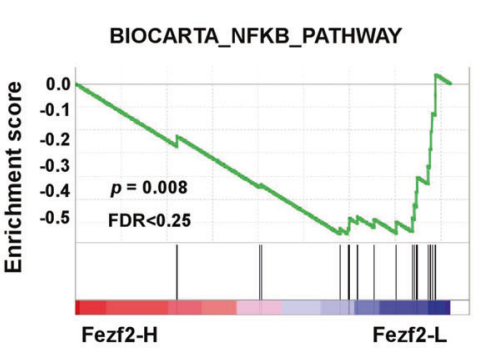

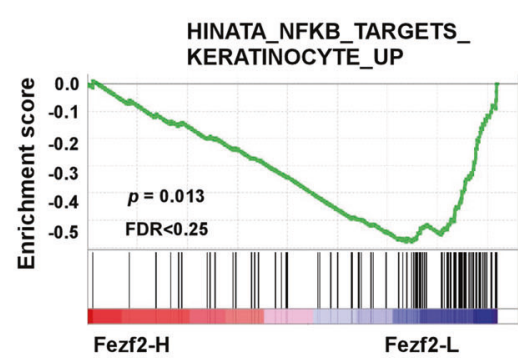

B

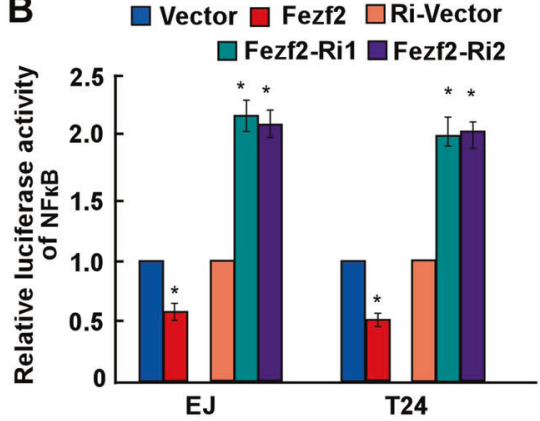

C

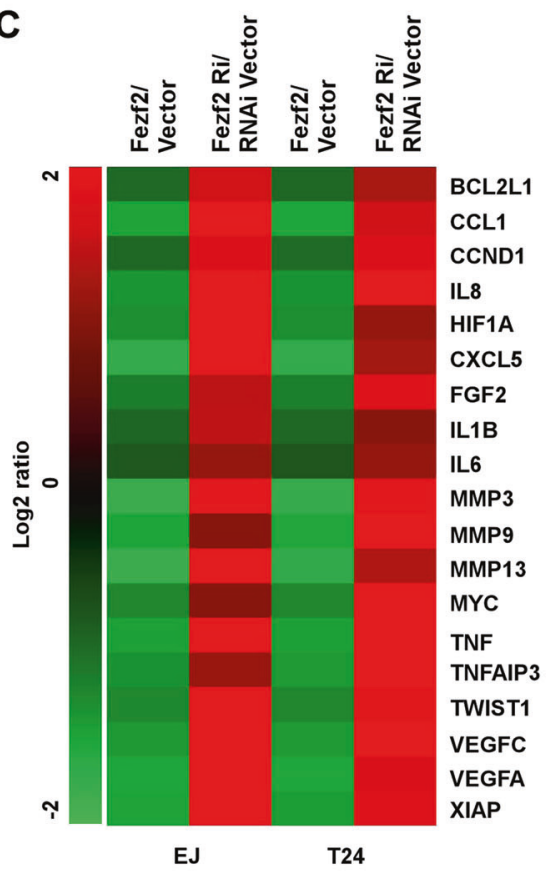

D

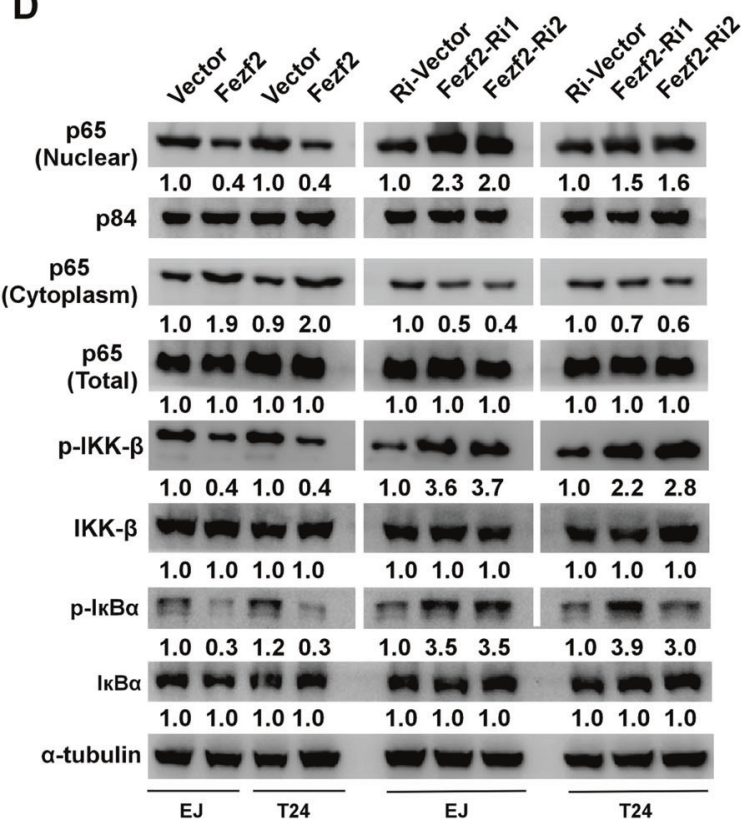

E

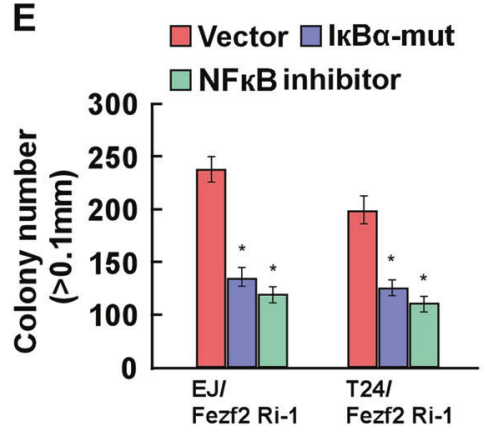

$\mathbf{F}$

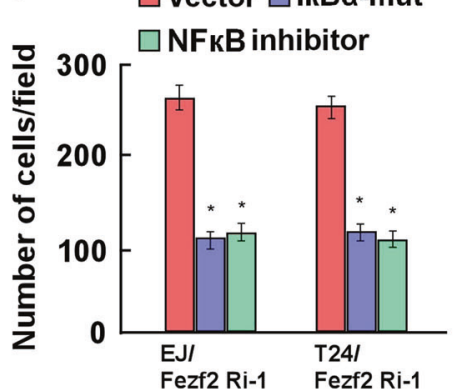

G

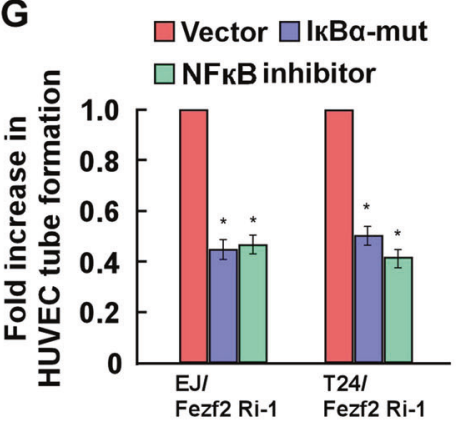

Fig. 5 Fezf2 regulates the NF- $\kappa B$ signaling pathway in bladder cancer. a GSEA plot showing Fezf2 expression is negatively correlated with NF- $\kappa \mathrm{B}$-activated gene signatures. b Analysis of luciferase reporter activity in indicated cells after transfection with pNF- $\kappa \mathrm{B}-$ luc plasmids or control-luciferase plasmid. c Real-time PCR analysis of NF- $\mathrm{KB}$ signaling pathway downstream gene expression in indicated cells. d Western blotting analysis of expression levels of indicated proteins in indicated cells. $\alpha$-tubulin was used as a loading control. e
Quantification of colony numbers in anchorage-independent growth assay. Colonies larger than $0.1 \mathrm{~mm}$ in diameter were scored. f Quantification (right panel) of invading cells analyzed with a transwell Matrigel assay. g Quantification of tubule formation by HUVECs cultured in Matrigel-coated plates with conditioned media from bladder cancer cells transfected with the vector I $\mathrm{K} B \alpha$-mut, or treated with the NF- $\kappa B$ inhibitor JSH-23. Each bar represents the mean \pm SD of three independent experiments. $* P<0.05$ 
A

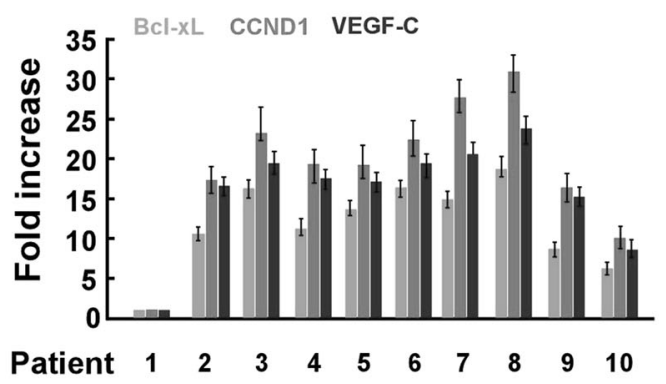

p65

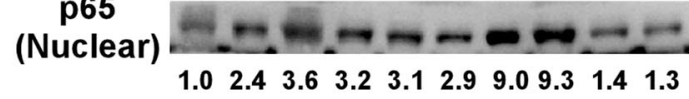

p84

Fezf2

$\begin{array}{lllllllllll}1.0 & 0.5 & 0.2 & 0.4 & 0.3 & 0.4 & 0.2 & 0.2 & 0.4 & 0.4\end{array}$

a-tubulin
B

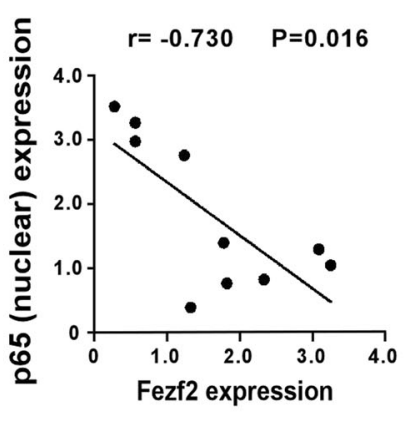

$r=-0.792 \quad P=0.006$

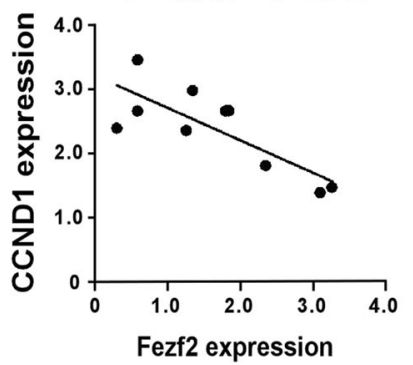

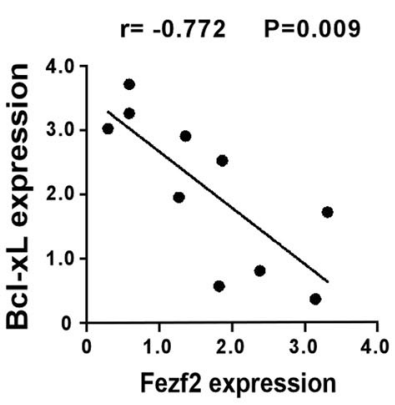

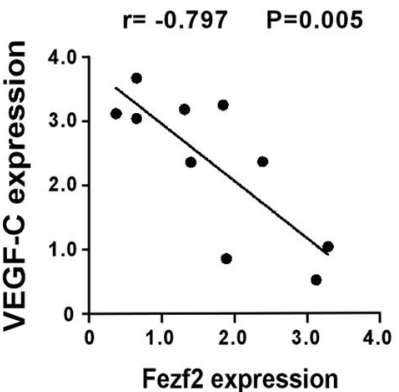

Fig. 6 Clinical relevance of Fezf2-mediated NF- $\mathrm{BB}$ activation in human bladder cancer. a Real-time PCR and Western blotting assays showing protein expression of Fezf2, BCL-xL, CCND1, VEGF-C, and nuclear p65 in 10 bladder cancer samples. $\alpha$-Tubulin and p84 were used as loading controls. b Correlation analysis of Fezf2 and BCL-xL, CCND1, VEGF-C, and nuclear p65 expression. Each bar represents the mean \pm SD of three independent experiments

expression produces the opposite effect through modulation of the NF-KB signaling pathway. These findings emphasize the prominent role of Fezf2 deregulation in promoting carcinogenesis and progression of bladder cancer.

Previous studies have shown that the NF-kB signaling pathway not only plays a pivotal role in immune and inflammatory responses, but is also essential for tumorigenesis $[14,15]$. Specific targeting of the NF- $\mathrm{KB}$ signaling pathway has been intensively explored for its potential as a treatment for a variety of malignancies [16, 17]. For example, inhibition of the NF- $\mathrm{BB}$ pathway by BAY 117085 , triptolide, or minnelide leads to deregulation of epithelial-mesenchymal transition (EMT) and in vitro neural invasion in pancreatic cancer [18]. In contrast, activation of NF- $\mathrm{kB}$ signaling by RPA2 overexpression promotes cell proliferation, adhesion, migration, and invasion, and induces EMT of breast cancer cells [19]. In addition, treatment with BMS-345541, an IKK inhibitor, significantly suppresses EMT and induces cell death in prostate cancer cells [20]. NF-KB signaling has also been shown to be critical in the oncogenesis and progression of bladder cancer. Cui et al. demonstrated that NF-kB promotes cell cycle progression and reduces apoptosis by upregulating survivin expression in bladder cancer cells [21]. Moreover, NF-кB signaling is involved in cisplatin-induced bladder cancer chemoresistance and tumor progresion [22]. These findings suggest that inhibition of NF- $\mathrm{KB}$ signaling is a potential therapeutic strategy for the treatment of bladder cancer.
Here we showed Fezf2 is downregulated in bladder cancer and its downregulation is correlated with poor clinical prognosis of bladder cancer patients. Overexpression of Fezf2 dramatically suppressed malignant phenotypic features including proliferation, migration, invasion, and angiogenesis through the inactivation of NF- $\mathrm{KB}$ signaling, suggesting that Fezf2 contributes to inactivation of NF- $\mathrm{KB}$ signaling and thereby represents a potential target for bladder cancer therapy.

Evidence suggests Fezf2 may regulate signaling pathways. For instance, overexpression of Fezf2 significantly increases the generation of rostral forebrain progenitors and inhibits the expression of transcription factors expressed by the midbrain and caudal diencephalon through the regulation of Wnt signaling [23]. In agreement with this study, Zhang et al. demonstrated that Fezf2 promotes neuronal differentiation through localized activation of $\mathrm{Wnt} / \beta$-catenin signaling during forebrain development [24]. In addition, activation of Fezf1 and Fezf2 genes by knockdown of Bre1a results in increased expression of the Notch signaling effector gene Hes5, and suppression of differentiation of neural precursor cells [25]. In nasopharyngeal carcinoma cells (NPCs), epigenetic silencing of Fezf2 may contribute to the perturbation of the $\mathrm{p} 53$ signaling pathway, which further promotes NPC cell growth and inhibits apoptosis [7]. The current study shows that Fezf 2 negatively regulates $\mathrm{NF}-\mathrm{\kappa B}$ signaling and tumorigenicity in bladder cancer. This is the first study demonstrating a novel linkage between 
Fezf2 and the NF-кB signaling pathway in cancer development and progression. However, the exact mechanism by which Fezf2 suppress the NF- $\mathrm{KB}$ signaling pathway remains unknown and warrants further investigation.

Fezf2 has been identified as a tumor suppressor, but its prognostic role in cancer has never been investigated. Our analysis has provided the first evidence that Fezf2 expression is closely correlated with clinical prognosis in bladder cancer. Patients with lower levels of Fezf2 expression in this study had shorter overall and recurrence-free survival time compared to patients with higher Fezf2 expression. In agreement with our results, Sheu et al. reported that chromosome 3p12.3-p14.2 deletion is a prognostic marker for advanced NPC patients [26]. These studies support the concept that Fezf2 is a novel predictor of prognosis in human cancers.

Our study has several limitations including the retrospective design and a relatively small sample size. A prospective study with a larger participant population will be required to conclusively demonstrate the prognostic value of Fezf 2 in bladder cancer patients. Moreover, much longer follow up will be necessary to draw definitive conclusions regarding the prognostic value of Fezf2. In addition, we did not evaluate lymph node status as a clinical variable that may be related to Fezf2 expression. This study included a heterogeneous group of patients in regard to disease stage. As such in patients with MIBC, the post-operative lymph node status could be evaluated, while for non-MIBC patients (without lymph node dissection), lymph node status could not be determined pathologically. Further studies are needed to evaluate correlation Fezf2 and lymph nodes metastasis in bladder cancer patients.

The present study demonstrated Fezf2 has an important role in bladder cancer progression through regulation of the NF- $\mathrm{BB}$ pathway, and our findings suggest that Fezf2 is a potential prognostic marker for bladder cancer patients. Fezf2 inhibitors have potential in therapeutic strategies for treatment of bladder cancer.

Acknowledgements We would like to thank Dr Michael A. McNutt (Beijing University Health Science Center, P. R. China) and Dr. Catherine R. Miller (University of Texas Medical Branch) for their assistance in editing this manuscript, and thank Dr Ping Yin (Huazhong University of Science and Technology) for his assistance in statistical analysis.

\section{Compliance with ethical standards}

Conflict of interest The authors declare that they have no conflict of interest.

\section{References}

1. Torre LA, Bray F, Siegel RL, et al. Global cancer statistics, 2012. CA Cancer J Clin. 2015;65:87-108.
2. Bellmunt J, Orsola A, Leow JJ, et al. Bladder cancer: ESMO practice guidelines for diagnosis, treatment and follow-up. Ann Oncol. 2014;25(Suppl 3):iii40-48.

3. Shimizu T, Hibi M. Formation and patterning of the forebrain and olfactory system by zinc-finger genes Fezf1 and Fezf2. Dev Growth Differ. 2009;51:221-31.

4. Hirata T, Nakazawa M, Muraoka O, et al. Zinc-finger genes Fez and Fez-like function in the establishment of diencephalon subdivisions. Development. 2006;133:3993-4004.

5. Yang N, Dong Z, Guo S. Fezf2 regulates multilineage neuronal differentiation through activating basic helix-loop-helix and homeodomain genes in the zebrafish ventral forebrain. J Neurosci. 2012;32:10940-8.

6. Zuccotti A, Le Magueresse C, Chen M, et al. The transcription factor Fezf2 directs the differentiation of neural stem cells in the subventricular zone toward a cortical phenotype. Proc Natl Acad Sci USA. 2014;111:10726-31.

7. Shu XS, Li L, Ji M, et al. FEZF2, a novel 3p14 tumor suppressor gene, represses oncogene EZH2 and MDM2 expression and is frequently methylated in nasopharyngeal carcinoma. Carcinogenesis. 2013;34:1984-93.

8. Gan Y, Chen D, Li X. Proteomic analysis reveals novel proteins associated with progression and differentiation of colorectal carcinoma. J Cancer Res Ther. 2014;10:89-96.

9. Denkert C, Budczies J, Kind T, et al. Mass spectrometry-based metabolic profiling reveals different metabolite patterns in invasive ovarian carcinomas and ovarian borderline tumors. Cancer Res. 2006;66:10795-804.

10. Yang $\mathrm{C}$, Zhang W, Wang L, et al. Musashi-2 promotes migration and invasion in bladder cancer via activation of the JAK2/STAT3 pathway. Lab Invest. 2016;96:950-8.

11. Subramanian A, Tamayo P, Mootha VK, et al. Gene set enrichment analysis: a knowledge-based approach for interpreting genome-wide expression profiles. Proc Natl Acad Sci USA. 2005;102:15545-50.

12. Cai J, Feng D, Hu L, et al. FAT4 functions as a tumour suppressor in gastric cancer by modulating Wnt/beta-catenin signalling. Br J Cancer. 2015;113:1720-9.

13. Han CP, Lee MY, Tzeng SL, et al. Nuclear Receptor Interaction Protein (NRIP) expression assay using human tissue microarray and immunohistochemistry technology confirming nuclear localization. J Exp Clin Cancer Res. 2008;27:25.

14. Hoesel B, Schmid JA. The complexity of NF-kappaB signaling in inflammation and cancer. Mol Cancer. 2013;12:86.

15. Wang L, Zhou W, Zhong Y, et al. Overexpression of G proteincoupled receptor GPR87 promotes pancreatic cancer aggressiveness and activates NF-kappaB signaling pathway. Mol Cancer. 2017;16:61.

16. Wang Y, Lin Z, Sun L, et al. Akt/Ezrin Tyr353/NF-kappaB pathway regulates EGF-induced EMT and metastasis in tongue squamous cell carcinoma. Br J Cancer. 2014;110:695-705.

17. Su X, Wang J, Chen W, et al. Overexpression of TRIM14 promotes tongue squamous cell carcinoma aggressiveness by activating the NF-kappaB signaling pathway. Oncotarget. 2016;7:9939-50.

18. Nomura A, Majumder K, Giri B, et al. Inhibition of NF-kappa B pathway leads to deregulation of epithelial-mesenchymal transition and neural invasion in pancreatic cancer. Lab Invest. 2016;96:1268-78

19. Chen CC, Juan CW, Chen KY, et al. Upregulation of RPA2 promotes NF-kappaB activation in breast cancer by relieving the antagonistic function of menin on NF-kappaB-regulated transcription. Carcinogenesis. 2017;38:196-206.

20. Ping $\mathrm{H}$, Yang F, Wang $\mathrm{M}$, et al. IKK inhibitor suppresses epithelial-mesenchymal transition and induces cell death in prostate cancer. Oncol Rep. 2016;36:1658-64. 
21. Cui X, Shen D, Kong C, et al. NF-kappaB suppresses apoptosis and promotes bladder cancer cell proliferation by upregulating survivin expression in vitro and in vivo. Sci Rep. 2017; 7:40723.

22. Sun Y, Guan Z, Liang L, et al. NF-kappaB signaling plays irreplaceable roles in cisplatin-induced bladder cancer chemoresistance and tumor progression. Int J Oncol. 2016;48:225-34.

23. Wang ZB, Boisvert $\mathrm{E}$, Zhang $\mathrm{X}$, et al. Fezf2 regulates telencephalic precursor differentiation from mouse embryonic stem cells. Cereb Cortex. 2011;21:2177-86.
24. Zhang S, Li J, Lea R, et al. Fezf2 promotes neuronal differentiation through localised activation of Wnt/beta-catenin signalling during forebrain development. Development. 2014;141:4794-805.

25. Ishino $Y$, Hayashi $Y$, Naruse $M$, et al. Brela, a histone $\mathrm{H} 2 \mathrm{~B}$ ubiquitin ligase, regulates the cell cycle and differentiation of neural precursor cells. J Neurosci. 2014;343067-78.

26. Sheu JJ, Lee CH, Ko JY, et al. Chromosome 3p12.3-p14.2 and 3q26.2-q26.32 are genomic markers for prognosis of advanced nasopharyngeal carcinoma. Cancer Epidemiol Biomark Prev. 2009;18:2709-16. 